\title{
Mathematical Simulation of the Near-Bottom Section of an Ascending Twisting Flow
}

\author{
S. P. Bautin ${ }^{a}$ and A. G. Obukhov ${ }^{b}$ \\ ${ }^{a}$ Ural State University of Railway Transport, ul. Kolmogorova 66, Yekaterinburg, 620034 Russia \\ ${ }^{b}$ Tyumen State Oil and Gas University, ul. Volodarskogo 38, Tyumen, 625000 Russia
}

Received September 4, 2012

\begin{abstract}
The available results of laboratory experiments on the formation of free vortices and controlling of their behavior are compared with the results of mathematical simulation of corresponding flows. This is accomplished by constructing solutions for a set of gas dynamics equations. The comparison is performed for a specific scheme of origination and functioning of free ascending twisting flows. In particular, it is shown that the experimental results confirm the proposed scheme of the origination and initial twisting of ascending vortex flows and validate the reason of their stable functioning with the help of the method intended for controlling generated vortices using vertical grids which was implemented in the experiments. The fact of origination of an ascending flow twisting and its directing is mathematically substantiated using the solution to a specific initially edge problem for a set of gas dynamics equations. A stationary flow whose parameters are close to gas-dynamic parameters of free vortices reproduced in the experiments is calculated.
\end{abstract}

DOI: $10.1134 /$ S0018151X1302003X

\section{INTRODUCTION}

The very interesting phenomena of ascending twisting flows (ATFs) of air are very often encountered in nature. Tornados and tropical cyclones serve as examples of such flows (see [1-3]). In spite of the fact that a large number of researchers have been investigating the ATF problem for decades (for a detailed bibliography, see, e.g., [3]), their studies still lack a sufficiently convincing theory that explains the reasons of origination, functioning, and natural disappearance of such flows, verified by experimental methods and adequate mathematical simulation.

A specific scheme of the origination and stable functioning of ATFs is proposed in [4]. The initial motion upon ATF origination is the vertical ascending motion of hot air caused by local heating of land or water areas (and neighboring air masses) by solar energy.

The arrows in Fig. 1a show an ascending flow, while the bold lines mark a warm region of the Earth's surface. The observations show that tropical cyclones and tornadoes originate in the regions of the Earth where the surface and neighboring air masses are strongly heated. Ascending air volumes are interchanged with new ones that enter the region of the ascending flow from below (see Fig. 1b). This is how the near-bottom section of an ascending flow is formed.

The initial near-bottom motion along the Earth's surface is radial: from the periphery regions to the bottom of an ascending heat flow from all sides. In Fig. 2a, the initial radial motion in the $x O y$ plane is represented by velocity vectors $\mathbf{V}$ of individual gas particles, while

the shaded circle shows the region of an ascending heat flow.

Apart from the horizontal air motion, a circumferential flow, namely gas twisting, is generated in the formed near-bottom section under the action of the Coriolis force $(\mathrm{CF})$. This situation is illustrated in Fig. 2a, where $\mathbf{F}_{c}$ are the $C F$ vectors acting on the material particles that move with the velocities $\mathbf{V}$ toward the common center. It is known [5] that under the action of CFs each individually moving particle in the northern hemisphere deviates to the right from the direction of its motion; in the southern hemisphere, the deviation is to the left. This yields gas twisting in the moving continuum, namely in the positive direction in the northern hemisphere and in the negative direction in the southern hemisphere.

If an ascending flow and the air motion toward its bottom are preserved for a long time, the Earth's rotation will yield substantial twisting of the air in the

(a)

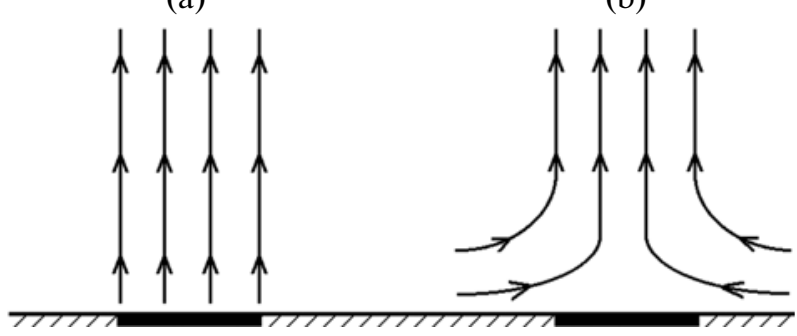

Fig. 1. Origination of a convective ascending flow. 


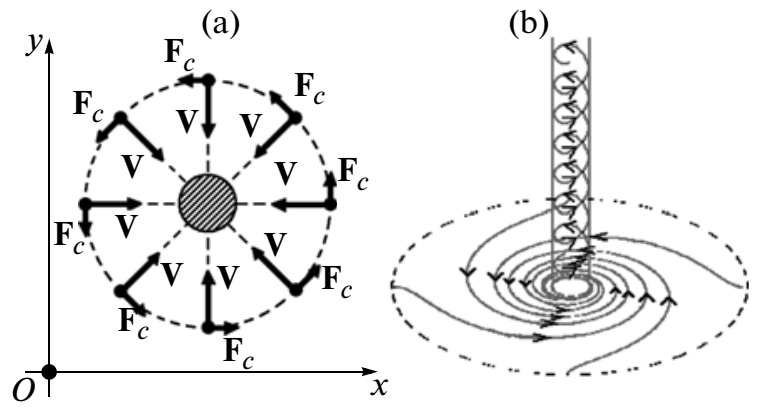

Fig. 2. The structure of a flow in the near-bottom and vertical sections of an ascending twisted flow.

near-bottom section of the ATF. This is the beginning of self-sustaining stable ATF functioning.

The energy required for this self-sustaining motion of air masses is supplied as follows (see Fig. 2b): (1) the Earth's rotation twists a gas in the near-bottom region; (2) gas twisting is transferred to the vertical section; (3) the centrifugal force creates a "tube with draft" in the vertical section, i.e., the pressure in the vicinity of its axis drops and there appears the effect of impenetrable walls since the air pressure in the vertical ATF section at its boundary coincides with the pressure of the external resting air; (4) the air (which is external with respect to the ATF) is pressed in the "tube with a draft" (where the pressure in the center is low) under the action of gravity.

The fact that twisting makes the flow in the vertical section stable is of principle significance. Note that the described scheme is in agreement with all hydrodynamic effects observed in tornados and tropical cyclones.

\section{EXPERIMENTAL RESULTS}

The process of ATF origination, the direction of its twisting, and the ATF structure proposed in [4] are confirmed by the experiments described in [3, 6-14].

In these experiments, a free vortex is generated above a metal table due to its heating from below. The term "free vortex" means that there is no forced twisting in the experiment, i.e., no convective flow generated due to heating, no initial air in the room, no table (before heating and in the process of heating). A free vortex predominantly twists in the positive direction (following the terminology of [3, 6-14], left-screw motion) since Moscow is in the northern hemisphere. The comparison of the experimental data with the data of field tornado observations and visualization of the free vortex motion reveals the identity of the experimental flows with those observed in nature; in particular, twisting of the air volumes adjacent from the inside to the outer boundary of the vertical sections is clearly fixed.
One of the main results of experiments [6-14] is to create conditions for the destruction of a vortex with vertical grids. The grid parameters are determined: size of cells, wire thickness, and the hight at which the destruction of the vortex with specific characteristics is most probable.

The experimentally ascertained fact of ATF destruction with a vertical grid, taking the proposed scheme of functioning of these flows into account [4], can be substantiated by the following gas dynamic considerations. In the presence of a solid vertical wall that rotates in the near-bottom section, the flow does not allow the vertical section of the vortex to approach the solid wall and makes the vortex either reflect at it or move in the parallel direction. If a grid obstacle blocks the vortex motion, the near-bottom vortex section passes through it almost without destruction. However, when the vertical section approaches the grid, the grid positioned at a tangent to the rotating vertical section hinders rotation and decelerates the circumferential component of the air velocity vector. Due to the decrease (or elimination) of twisting and "tube wall" damage, the flow stability in the vertical section decreases as well, and the external nonrotating air brings the vortex as a whole to a stop. The method for terminating the air motion in the ATF by damaging the "tube walls" is also proposed in the patent [15].

\section{MATHEMATICAL SIMULATION}

As distinct from the previous studies, the flows in the ATF are simulated in $[4,16,17]$ using a set of equations of gas dynamics since it most adequately describes an ideal gas flow. In particular, plain isentropic flows in the ATF near-bottom region are simulated with the solutions to the following set of equations with partial derivatives

$$
\left\{\begin{array}{l}
c_{t}+u c_{r}+\frac{(\gamma-1)}{2} c\left(u_{r}+\frac{u}{r}\right)=0, \\
u_{t}+u u_{r}-\frac{V^{2}}{r}+\frac{2}{(\gamma-1)} c c_{r}=a v, \\
v_{t}+u v_{r}+\frac{u v}{r}=-a u .
\end{array}\right.
$$

Here, $t$ is the time; $r$ is the distance to the origin in the $x O y$ plane; $c=\rho^{(\gamma-1) / 2}$ is the speed of sound; $\rho$ is the gas density; $\gamma=1.4$ is the adiabatic (polytrope) exponent of the air; $u, v$ are the radial and circumferential components of the gas velocity vector; $a=2 \Omega \sin \psi$, where $\Omega$ is the modulus of the Earth's angular rotation; and $\psi$ is the latitude of the point of the plane frame origin. In set (1), with the help of scaled values of velocity, speed of sound, time, and distance $\left(u_{00}, c_{00}, t_{00}, r_{00}\right)$, the following dimensionless variables were introduced in the standard manner: $f=$ $f_{*} / f_{00}$, where $f_{*}$ and $f_{00}$ are the dimensional and scaled 
values of $f$. In this case, it is assumed that $u_{00}=r_{00} / t_{00}$, $c_{00}=u_{00}$.

The beginning of formation of the ATF near-bottom region is simulated with solution of the following drain problem.

Let the resting gas whose speed of sound is equal to unity be at the instant $t=0$ to the right of the point $r=r_{0}=$ const $>0$. Starting from the instant $t=0$, the gas starts smoothly draining at the point $r=r_{0}$ in accordance with the assigned law

$$
\begin{gathered}
\left.u(t, r)\right|_{r=r 0}=u_{*}(t), \quad u_{*}(0)=0, \\
u_{* t}(0)=u_{* 1}=\mathrm{const}<0 .
\end{gathered}
$$

In this case, it is implied that, starting from the instant $t=0$, the air in the $0 \leq r \leq r_{0}$ starts moving upward, e.g., like an ascending convective flow. This, in turn, determines the negative radial velocity in the plain near-bottom flow.

For the solution to the drain problem to be constructed, it is necessary to join the desired flow through the acoustic characteristic

$$
C^{+}: r=t+r_{0}
$$

to the uniform resting gas.

Theorem: For problem (1), (2), with the side conditions

$$
\left.c(t, r)\right|_{C^{+}}=1,\left.\quad u(t, r)\right|_{C^{+}}=0,\left.\quad v(t, r)\right|_{C^{+}}=0
$$

there is a unique analytical solution, and the circumferential speed can be represented as follows:

$$
v(\xi, \varsigma)=-a u_{1}(\varsigma) \frac{\xi^{2}}{2}+\ldots,
$$

where $\xi=t-r+r_{0}, \varsigma=r-r_{0}$, and

$$
u_{1}(\varsigma)=\frac{1}{\sqrt{\varsigma+r_{0}}\left\{(\gamma+1)\left[\sqrt{r_{0}}-\sqrt{\varsigma+r_{0}}\right]+\frac{1}{u_{* 1} \sqrt{r_{0}}}\right\}}<0
$$$$
\text { at } \varsigma>0 \text {. }
$$

The proof of the theorem is not given here, since the key stages of this procedure repeat those of the theorem proof from [17].

It follows from formulas (3) and (4) that at $a>0$ (northern hemisphere) we have

$$
\left.v\right|_{C^{+}}=0,\left.\quad v_{r}\right|_{C^{+}}=0,\left.\quad v_{r r}\right|_{C^{+}}>0,
$$

For the southern hemisphere $(a<0)$, the sign "less" should be used in the last inequality. Thus, immediately at $t>0$, the gas flow formed in the process of smooth radial gas drain on the $r=r_{0}$ circumference starts twisting in some vicinity of the acoustic $C^{+}$characteristic under the action of the Coriolis force: in the positive and negative directions for the northern and southern hemispheres, respectively.

The gas-dynamic parameters of a stationary flow that is formed with time in the near-bottom region are determined in the process of the numerical solution of set (1), with the equality $\partial / \partial t=0$ taken into account. Figure 3 shows four streamlines of the stationary flow



Fig. 3. Isolated streamlines of the flow in the near-bottom region.

at the following entry parameters: $c_{00}=333 \mathrm{~m} / \mathrm{s}, r_{00}=$ $20 \mathrm{~m}, \sin \psi=0.866$, and $r_{0}=0.0012$, which corresponds to the dimensional value of $0.024 \mathrm{~m}$. In Fig. 3, a meter is taken as the unit of distance. The dimensional values of the radial and circumferential velocities of the drain circumference (i.e., at $r=r_{0}$ ) are $0.139 \mathrm{~m} / \mathrm{s}$ and $1.066 \mathrm{~m} / \mathrm{s}$, respectively. The obtained magnitudes correspond to the results of specific experiments considered in [14].

A specific streamline of the stationary flow is numerically reconstructed in the form of the $\varphi=\varphi(r)$ dependence when constructing a solution to the following Cauchy problem:

$$
\frac{d \varphi}{d r}=\frac{v^{o}}{r u^{o}} ; \varphi\left(r_{\text {in }}\right)=\varphi_{0} ; \quad \varphi_{0}=\text { const }
$$

where $u^{o}, v^{o}$ are the radial and circumferential components of the gas velocity vector in a stationary flow; $\varphi$ is the polar angle; and $r_{\text {in }}$ is the inflow radius. The differential equation in the Cauchy problem is a corollary fact of the following set of ordinary differential equations:

$$
\frac{d r}{d t}=u^{o}, \frac{d \varphi}{d t}=\frac{V^{o}}{r}
$$

The path of an individual gas particle is reconstructed upon the solution of this set of equations.

It should be noted that the density on the drain circumference turned out to be $\rho\left(r_{0}\right)=1-4.3 \times 10^{-6}$. This magnitude almost does not differ from the unit density of an unperturbed gas. However, if we perform a priori simulation of an incompressible fluid flow, the corresponding stationary problem (under the condition of satisfiability of the equation of state of the analyzed gas) lacks a solution.

\section{CONCLUSIONS}

The solution to the initial-edge problem for a set of gas dynamics equations which substantiates the fact of origination and direction of twisting of the ascending 
flow is derived. The near-bottom region of a stationary ascending flow whose parameters are close to the gasdynamic parameters of free vortices in available experiments has been calculated.

\section{ACKNOWLEDGMENTS}

This study was supported by the Russian Foundation for Basic Research, project no. 11-01-00198.

\section{REFERENCES}

1. Nalivkin, D.V., Hurricanes, Storms, and Tornadoes: Geographical Characteristics and Geological Activity, New York: Taylor and Francis, 1983.

2. Nalivkin, D.V., Smerchi (Tornadoes), Moscow: Nauka, 1984.

3. Varaksin, A.Yu., Romash, M.E., and Kopeitsev, V.N., Tornado (Tornado), Moscow: Fizmatlit, 2011.

4. Bautin, S.P., Tornado i sila Koriolisa (Tornado and Coriolis Force), Novosibirsk: Nauka, 2008.

5. Loitsyanskii, L.G. and Lur'e, A.I., Kurs teoreticheskoi mekhaniki (A Course of Theoretical Mechanics), Moscow: Nauka, 1983, vol. 2.

6. Varaksin, A.Yu., Romash, M.E., Kopeitsev, V.N., and Taekin, S.I., High Temp., 2008, vol. 46, no. 6, p. 888.
7. Varaksin, A.Yu., Romash, M.E., Taekin, S.I., and Kopeitsev, V.N., High Temp., 2009, vol. 47, no. 1, p. 78.

8. Varaksin, A.Yu., Romash, M.E., and Kopeitsev, V.N., High Temp., 2009, vol. 47, no. 6, p. 836.

9. Varaksin, A.Yu., Romash, M.E., Kopeitsev, V.N., and Taekin, S.I., High Temp., 2010, vol. 48, no. 2, p. 251.

10. Varaksin, A.Yu., Romash, M.E., and Kopeitsev, V.N., High Temp., 2010, vol. 48, no. 3, p. 411.

11. Varaksin, A.Yu., Romash, M.E., and Kopeitsev, V.N., High Temp., 2010, vol. 48, no. 4, p. 588.

12. Varaksin, A.Yu., Romash, M.E., Kopeitsev, V.N., and Gorbachev, M.A., High Temp., 2010, vol. 48, no. 6, p. 918.

13. Varaksin, A.Yu., Romash, M.E., Kopeitsev, V.N., and Gorbachev, M.A., High Temp., 2011, vol. 49, no. 2, p. 310 .

14. Varaksin, A.Yu., Romash, M.E., Kopeitsev, V.N., and Gorbachev, M.A., High Temp., 2012, vol. 50, no. 4, p. 496.

15. Bautin, S.P., Bautin, P.S., and Bautina, A.S., RF Patent no. 2407281 , Byull. Izobret., no. 36 (2010).

16. Bautin, S.P. and Obukhov, A.G., Matematicheskoe modelirovanie razrushitel'nykh atmosfernykh vikhrei (Mathematical Simulation of Destructive Atmospheric Vortexes), Novosibirsk: Nauka, 2012.

17. Bautin, S.P. and Krutova, I.Yu., High Temp., 2012, vol. 50 , no. 3 , p. 444. 